\title{
Brown-Vialetto-Van Laere and Fazio Londe syndrome is associated with a riboflavin transporter defect mimicking mild MADD: a new inborn error of metabolism with potential treatment
}

\author{
Annet M. Bosch • Nico G. G. M. Abeling • Lodewijk IJlst • Hennie Knoester • \\ W. Ludo van der Pol • Alida E. M. Stroomer • Ronald J. Wanders • Gepke Visser • \\ Frits A. Wijburg • Marinus Duran • Hans R. Waterham
}

Received: 13 September 2010 /Revised: 20 October 2010 / Accepted: 26 October 2010 /Published online: 26 November 2010

(C) The Author(s) 2010. This article is published with open access at Springerlink.com

\begin{abstract}
We report on three patients (two siblings and one unrelated) presenting in infancy with progressive muscle weakness and paralysis of the diaphragm. Metabolic studies revealed a profile of plasma acylcarnitines and urine organic acids suggestive of a mild form of the multiple acyl-CoA
\end{abstract}

Communicated by: K. Michael Gibson

Marinus Duran and Hans R. Waterham contributed equally

Competing interests: None declared.

A. M. Bosch $\cdot$ H. Knoester $\cdot$ F. A. Wijburg

Department of Pediatrics, Academic Medical Center,

University of Amsterdam,

Amsterdam, The Netherlands

N. G. G. M. Abeling • L. IJlst • A. E. M. Stroomer •

R. J. Wanders $\cdot$ M. Duran $\cdot$ H. R. Waterham

Laboratory Genetic Metabolic Diseases,

Academic Medical Center, University of Amsterdam,

Amsterdam, The Netherlands

W. L. van der Pol

Rudolf Magnus Institute of Neuroscience, Department of Neurology and Pediatric Neuromuscular Center 'Spieren Voor Spieren',

University Medical Center Utrecht,

Utrecht, The Netherlands

G. Visser

Department of Metabolic Diseases,

Wilhelmina Children's Hospital,

Utrecht, The Netherlands

A. M. Bosch $(\square)$

Department of Pediatrics, Academic Medical Center (H7 270),

University Hospital of Amsterdam,

Meibergdreef 91105 AZ,

Amsterdam, The Netherlands

e-mail: a.m.bosch@amc.nl dehydrogenation defect (MADD, ethylmalonic/adipic acid syndrome). Subsequently, a profound flavin deficiency in spite of a normal dietary riboflavin intake was established in the plasma of all three children, suggesting a riboflavin transporter defect. Genetic analysis of these patients demonstrated mutations in the C20orf54 gene which encodes the human homolog of a rat riboflavin transporter. This gene was recently implicated in the Brown-Vialetto-Van Laere syndrome, a rare neurological disorder which may either present in infancy with neurological deterioration with hypotonia, respiratory insufficiency and early death, or later in life with deafness and progressive ponto-bulbar palsy. Supplementation of riboflavin rapidly improved the clinical symptoms as well as the biochemical abnormalities in our patients, demonstrating that high dose riboflavin is a potential treatment for the Brown-Vialetto-Van Laere syndrome as well as for the Fazio Londe syndrome which is considered to be the same disease entity without the deafness.

\section{Introduction}

Brown-Vialetto-van Laere syndrome (MIM 211530), is a rare neurological disorder which may present in infancy with a devastating neurological deterioration with hypotonia, respiratory insufficiency and early death, or later in life with deafness and progressive ponto-bulbar palsy (Green et al. 2010; Sathasivam 2008). The same clinical presentation without deafness is also known as Fazio Londe disease (MIM 211500). Both are now considered to be a single disease entity (Dipti et al. 2005).

The multiple acyl-CoA dehydrogenation defect (MADD) is an inherited defect of mitochondrial fatty acid beta-oxidation 
and branched-chain amino acid catabolism (MIM231680). This disorder is characterized by the malfunctioning of an array of dehydrogenation reactions caused by the absence of either electron transfer flavoprotein (ETF) or electron transfer flavoprotein oxidoreductase (ETFDH). The electrons which are liberated in the dehydrogenation reactions are primarily captured by flavin adenine dinucleotide (FAD), a metabolite of the vitamin riboflavin. MADD may present at any age, varying from a lethal neonatal form and an infantile form characterized by hazardous hypoketotic hypoglycaemia (Rhead et al. 1987) to relatively mild adult lipid storage myopathy (Antozzi et al. 1994). Riboflavin responsive forms of MADD are usually the consequence of a defective ETFDH (Olsen et al. 2007).

We present two siblings and one unrelated patient, presenting in infancy with progressive muscle weakness and paralysis of the diaphragm, later recognized as Fazio Londe and Brown-Vialetto-van Laere syndrome. Our patients demonstrated abnormalities on metabolic evaluation mimicking mild MADD and were found to be severely flavin deficient. Riboflavin therapy resulted in a rapid clinical and biochemical improvement.

\section{Patients and methods}

\section{Patient 1}

A 6 month old boy, the first child of healthy parents, presented with a short history of progressive muscle weakness followed by life threatening apnoeic spells necessitating artificial ventilation. Neurological examination revealed generalized muscle weakness with a severe head lag. A complete diaphragmatic paralysis was detected on ultrasound. Spinal muscular atrophy (SMA) and SMA with respiratory distress (SMARD) were excluded by genetic testing. Muscle histology at the age of 7 months demonstrated unclassified myopathic abnormalities. Biochemical analysis of the mitochondrial respiratory chain in fresh muscle tissue gave inconclusive results. Metabolic studies revealed abnormal concentrations of short- and medium-chain plasma acylcarnitines and a urine organic acid profile was suggestive of MADD (Tables 1 and 2). Because of the possibility of riboflavin-responsiveness (Olsen et al. 2007), the patient was treated with high dose oral riboflavin (vitamin B2, $10 \mathrm{mg} / \mathrm{kg}$ body weight/day). The MADD associated metabolic abnormalities disappeared within days. Cessation of riboflavin supplementation resulted in a recurrence of the abnormal metabolic profile in spite of a normal dietary riboflavin intake, and riboflavin medication was restarted. Classical MADD was ruled out by sequence analysis of the ETFDH, ETFA and ETFB genes. The biochemical profile, absence of urine thiosulfate and sulfo(thio)cysteine (Duran et al. 1997) argued against an ethylmalonic syndrome (ETHE) (Tiranti et al. 2009). Therefore we hypothesized a decreased availability of flavin adenine dinucleotide (FAD), the cofactor of MADD, due to defective uptake, synthesis or transport, to be the underlying cause of the disease.

A tracheotomy was placed and the boy's muscle tone slowly improved over the next month. He was able to walk independently at the age of 22 months. As the diaphragmatic paralysis persisted, he needed nightly ventilation until the age of 41 months. Recently, the clinical diagnosis of Fazio Londe Syndrome was made. He is currently 46 months old, his cognitive development is fully normal and he demonstrates no further cranial nerve palsy.

\section{Patient 2}

Recently, the 3 months-old sister of patient 1 presented with failure to thrive and generalized axial muscle weakness. Based on the results of the additional studies in her brother, flavin deficiency was suspected, subsequently confirmed by

Table 1 Plasma acylcarnitines ( $\mu \mathrm{mol} / \mathrm{L}$ ) in patient, 2, and 3 compared to a MADD patient, a patient with the ethylmalonic acid syndrome, and healthy controls. Bold values are outside normal range

\begin{tabular}{|c|c|c|c|c|c|c|}
\hline & Pat 1 before riboflavin & Pat 2 before riboflavin & Pat 3 at start of treatment & MADD & ETHE & Controls \\
\hline $\mathrm{C} 0$ & 18.3 & 35.1 & 37.6 & 4.2 & 35.4 & $22-55$ \\
\hline $\mathrm{C} 2$ & 3.2 & 4.0 & 0.74 & 1.9 & 5.1 & $3.4-13$ \\
\hline $\mathrm{C} 4$ & 0.65 & 2.97 & 0.43 & 8.55 & 2.07 & $0.07-0.58$ \\
\hline $\mathrm{C} 5$ & 0.29 & 1.99 & 0.91 & 0.31 & 0.32 & $0.04-0.22$ \\
\hline C6 & 0.27 & 0.87 & 0.02 & 0.14 & 0.05 & $0.02-0.12$ \\
\hline $\mathrm{C} 8$ & 0.18 & 0.70 & 0.53 & 0.25 & 0.04 & $0.04-0.22$ \\
\hline $\mathrm{C} 10$ & 0.25 & 0.86 & 0.77 & 0.49 & 0.06 & $0.04-0.30$ \\
\hline $\mathrm{C} 12$ & 0.15 & 0.44 & 0.64 & 0.49 & 0.05 & $0.04-0.12$ \\
\hline $\mathrm{C} 14$ & 0.14 & 0.23 & 0.46 & 0.39 & & $<0.08$ \\
\hline $\mathrm{C} 16$ & 0.19 & 0.19 & 0.32 & 0.37 & 0.09 & $0.06-0.24$ \\
\hline C18:1 & 0.16 & 0.13 & 0.42 & 0.53 & 0.16 & $0.06-0.28$ \\
\hline
\end{tabular}


Table 2 Urine organic acids ( $\mathrm{mmol} / \mathrm{mol}$ creat) in patient 1 compared to a MADD patient, a patient with the ethylmalonic acid syndrome, and healthy controls

\begin{tabular}{lllll}
\hline & Pat 1 before riboflavin & MADD & ETHE & Controls \\
\hline EMA & 62 & 2648 & 48 & $<15$ \\
Glutarate & 1 & 19986 & 1 & $<15$ \\
Adipate & 70 & 5207 & 2 & $2-26$ \\
2-OH-glutarate & 106 & 832 & 19 & $11-51$ \\
Suberate & 26 & 764 & 1 & $<15$ \\
Hexanoylglycine & + & ++ & - & \\
Isovalerylglycine & + & ++ & + & \\
\hline
\end{tabular}

the analysis of plasma flavins and acylcarnitines, and treatment with riboflavin (10 $\mathrm{mg} / \mathrm{kg}$ body weight /day) was started. This resulted in normalization of her muscle tone within 7 days and a rapid catch-up growth. Sensorineural hearing loss was excluded by brainstem evoked response audiometry. Therefore, the clinical diagnosis of Fazio Londe Syndrome was made. After 3 months of riboflavin supplementation her growth and development are normal.

\section{Patient 3}

A now 7 year old girl, second child of healthy parents, demonstrated feeding problems and a slow motor development since birth. At the age of 5 months she had generalized weakness, more pronounced in distal than proximal muscle groups, and developed respiratory insufficiency necessitating artificial ventilation due to diaphragmatic paralysis. Furthermore, she was found to have a severe sensorineural hearing loss. SMA and SMARD were excluded by genetic testing. Muscle histology at the age of one year showed slight predominance of type 1 fibers without type grouping. Biochemical analysis of the mitochondrial respiratory chain in muscle tissue was inconclusive.

At the age of 6 months, metabolic studies revealed an acylcarnitine profile suggestive of MADD (Table 1). A fat restricted diet and supplementation with carnitine $(50 \mathrm{mg} /$ $\mathrm{kg} /$ day), riboflavin (10 mg/kg /day) and glycine and 3hydroxybutyrate according to the publication by Van Hove et al. (2003) were started. Her muscle strength improved and from the age of 2 years artificial ventilation was only necessary during sleep. Cognitive development was normal.

Classical MADD was ruled out by enzymatic testing in fibroblasts and mutation analysis of the ETFDH, ETFA and ETFB genes. Following the normalization of the acylcarnitine profiles, the fat restriction was gradually discontinued and the glycine, 3-hydroxybutyrate and carnitine supplementations were stopped without problems. However, withdrawal of riboflavin at the age of 4 years resulted in a rapid clinical deterioration with vomiting, progressive fatigue, and elevations of lactate, liver enzymes and CK. The acylcarnitine profile became abnormal again. Reintroduction of riboflavin (50 mg b.i.d.) resulted in clinical improvement and normal- ization of the biochemical abnormalities. Sequence analysis of the mitochondrial FAD transporter (Spaan et al. 2005) was performed on suspicion of a possible mitochondrial riboflavin transporter defect, but showed no abnormalities.

After reintroduction of riboflavin supplementation the patient had a stable clinical course. She walked with a foot drop and attended a school for hearing impaired children. The parents gradually decreased the dose of riboflavin to $10 \mathrm{mg} \mathrm{b}$. i.d. because they suspected gastrointestinal side effects of the high doses of riboflavin. However, after 5 years she developed palsies of the 7 th and $12^{\text {th }}$ cranial nerves, and became wheelchair bound. Furthermore, following a lower respiratory tract infection at the age of 6.5 years she became once again completely ventilator dependent, and her muscle strength slowly deteriorated. Only recently, the clinical diagnosis of Brown-Violetto-Van Laere syndrome was confirmed. The riboflavin dose was increased to $50 \mathrm{mgt.i.d}$., thus far without improvement of her symptoms.

\section{Methods}

Selective screening for inborn errors of metabolism was performed by standard techniques including analysis of organic acids in urine and acylcarnitines in plasma. Plasma riboflavin, flavin mononucleotide (FMN) and FAD concentrations were measured by high-performance liquid chromatography using fluorescence detection of the analytes according to the method of Capo-chichi et al. (2000) with minor modifications.

Because plasma flavins were deficient in all three patients despite a normal intake, we suspected a defect in intestinal riboflavin uptake and we therefore performed mutation analysis of the $C 20$ orf 54 gene. The protein encoded by this gene is highly similar to the rat rRFT2 protein, which has been shown to be the transporter of riboflavin in the small intestine (Yamamoto et al. 2009). Mutation analysis of the gene was performed by sequencing all exons plus flanking intronic sequences amplified from genomic DNA extracted from the patients' cells. Confirmation of the mutation in the parental genes was performed in genomic DNA extracted from blood cells.

Fibroblast fatty acid oxidation studies were carried out in patients 1 and 3 by incubating the cells with $\mathrm{U}_{-}{ }^{13} \mathrm{C}$ palmitate 
for 96 hours followed by the analysis of acylcarnitines in the supernatant (Ventura et al. 1999).

After the diagnosis had been established, the acylcarnitine profiles of the newborn screening bloodspots of patients 1 and 2 were evaluated by standard ESI-tandem mass spectrometric analysis. The newborn sample of patient 3 was no longer available.

\section{Results}

The initial diagnostic work-up of patient 1 included an analysis of plasma acylcarnitines and urine organic acids (Tables 1 and 2). A moderate accumulation of short and medium-chain acylcarnitines was observed. The long-chain acylcarnitines were normal. The profiles observed in patients 2 and 3 were similar but differed somewhat in the levels of the long-chain acylcarnitines, demonstrating the biochemical variability of this defect. The acylcarnitine profiles of the newborn screening bloodspots of patients 1 and 2 were normal (not shown), which demonstrates that newborn screening for a riboflavin transporter by this method is not feasible.

For comparison, plasma of a patient with an ETHE defect was also investigated, demonstrating an accumulation of short-chain acylcarnitines only (Table 1). Finally, plasma of an infantile patient with hypoketotic hypoglycaemic MADD resulting from a homozygous mutation of the ETFDH gene showed increased levels of all characteristic short-, medium-, and long-chain acylcarnitines as well as glutarylcarnitine. Glutarylcarnitine was normal in both the riboflavin transporter patients and the ETHE patient (Table 1). The C4 carnitine consisted mainly of the isobutyrylcarnitine, pointing to a considerable vulnerability of Acad 8 in all conditions described here.

Organic acid analysis of the urine of patient 1 demonstrated a pattern resembling that of mild MADD (Table 2). Short and branched chain acylglycines were clearly elevated as was the D-2-hydroxyglutaric acid. The ETHE patient had a comparable excretion pattern, although hexanoyl-glycine appeared to be normal. Patient 2 and 3 were not investigated. Neither the riboflavin transporter patient nor the ETHE patient had an increased urine glutaric acid, in contrast to the ETFDH patient (Table 3).

The fibroblast fatty acid oxidation studies in patients 1 and 3 revealed a normal profile of acylcarnitines, in sharp contrast to the results obtained in the ETFDH patient, showing accumulation of all C4-C16 intermediates.

Concentrations of riboflavin, FMN and FAD in plasma before treatment revealed a deficiency of all flavins in patients 1 and 2 (Table 3 ) whereas patient 3 had markedly decreased levels of FMN and FAD. Riboflavin levels normalized within weeks after the start of riboflavin supplementation. Cessation of supplementation in patients 1 and 3 resulted in rapid recurrence of the deficient state. Plasma flavins of the mother of patients 1 and 2 were normal.

Patient 1 and 2 were found to be homozygous for a pathogenic splice acceptor site mutation in C20orf54: c.1198$2 \mathrm{~A}>\mathrm{C}$ which is most probably pathogenic as it concerns a mutation of an invariant nucleotide of a splice acceptor site and thus will lead to incorrect mRNA splicing. The parents, second cousins, were heterozygous for this mutation. Patient 3 was found to be heterozygous for two mutations in the C20orf54 gene: c.49T $>C$ (p.W17R) and c.639C $>\mathrm{G}$ (p.Y213X). The latter mutation was also identified by Green et al. (2010). The c.49T $>$ C mutation affects a tryptophan at position 17 , which is highly conserved in orthologs of different species.

\section{Discussion}

Riboflavin is the precursor of FAD, which acts as an electron acceptor in a number of acyl-CoA dehydrogenation reactions involved in mitochondrial fatty acid oxidation and branchedchain amino acid catabolism (Gregersen et al. 2008). An inherited or acquired deficiency of riboflavin will therefore mimic the biochemical presentation observed in classical MADD, which was indeed observed in a riboflavin-deficient rat (Goodman 1981). The biochemical abnormalities observed in our patients are reminiscent of the mild forms of MADD, originally described as ethylmalonic/adipic aciduria (Tanaka et

Table 3 Plasma flavin values in patients 1, 2,and 3 before and after the start of riboflavin therapy

\begin{tabular}{llll}
\hline & Riboflavin (nmol/l) & FMN (nmol/l) & FAD (nmol/l) \\
\hline Controls (mean +/- 2 SD) (n=43, ages 0-35 y) & $3.9-49$ & $2.8-11.4$ & $46-114$ \\
Patient 1 before treatment & 1.4 & 1.7 & 31 \\
Patient 12 weeks after start therapy & 18.5 & 2.1 & 100 \\
Patient 2 before treatment & $<1$ & $<1$ & 30 \\
Patient 24 weeks after start treatment & 46.0 & 4.0 & 70 \\
Patient 3 at start of treatment & 7.6 & $<1$ & 24.5 \\
Patient 3 with supplementation & 12.3 & 8.3 & 105 \\
\hline
\end{tabular}


al. 1977). Our data suggest that the short-chain and mediumchain acyl-CoA dehydrogenases are more vulnerable to the shortage of the physiological electron acceptor than the longchain analogues. Similarly, glutaryl-CoA dehydrogenase was only mildly affected, in contrast to the D-2 hydroxyglutarate dehydrogenase.

Based on the profiles of acylcarnitines and organic acids it is difficult to make the distinction between the riboflavin transporter defect and the ETHE defect. Both conditions, however, have their own diagnostic analytes, i.e., riboflavin in the riboflavin transporter defect (Brown-Vialetto-Van Laere / Fazio Londe syndrome) and thiosulfate and sulfo (thio)cysteine in ETHE (Duran et al. 1997). Metabolic studies in intact fibroblasts were normal in the transporter defect cells, probably because the riboflavin in the cell culture medium corrected the defect resulting in a normal fatty acid oxidation. Likewise, maternal riboflavin supply is the probable cause of the normal clinical condition of the patients at birth and the fact that the acylcarnitines were normal in the newborn blood spots of patients 1 and 2 .

Riboflavin deficiency usually presents with different clinical symptoms (Powers 2003), but neurological symptoms resolving with riboflavin supplementation have been previously reported in a child with moderate riboflavin deficiency (Leshner 1981). Although further pathophysiological studies are needed to explain the clinical symptoms, we demonstrate that the riboflavin deficiency in our patients resulted from a defect in the riboflavin transporter, encoded by the C20orf54 gene.

Remarkably, at the same time that we discovered the molecular defects in our patients, causing a defective intestinal riboflavin transport, Green et al. (2010) reported the identification of mutations in the C20orf54 gene as the cause of the Brown-Vialetto-Van Laere Syndrome (MIM 211530). Indeed, the clinical presentation of all three patients is compatible with the diagnosis of BrownVialetto-van Laere (patient 3) or the Fazio Londe (patients 1 and 2) syndrome. This implies that at least part of the clinical signs and symptoms observed in these syndromes are caused by a deficiency of riboflavin and subsequently of FAD and FMN. The striking clinical and biochemical improvement on riboflavin supplementation seen in our patients strongly supports this hypothesis. We therefore presume that riboflavin may be an effective therapy in the Brown-Vialetto-Van Laere syndrome, at least in young patients. Early treatment appears to be crucial as diaphragmatic paralysis may be irreversible. Furthermore, in spite of the riboflavin supplementation since the age of 6 months, patient three now, at age 7 , demonstrates the neurological deterioration frequently observed in untreated BrownVialetto-Van Laere patients. This can be due to damage which already occurred during the extended periods of low FAD values probably present during the first 6 months of life as well as around the age of four, or to periods of limited riboflavin availability during intercurrent illnesses, as she demonstrated clinical deterioration mostly during periods of viral illness. On the other hand, it is possible that riboflavin supplementation only shifts the clinical course of the Brown-Vialetto-Van Laere / Fazio Londe syndrome to a later presentation of the clinical symptoms. A long term follow up of a cohort of early treated children, and more insight in the pathophysiology is warranted.

Finally, our results demonstrate that selective metabolic screening, including acylcarnitine profiling and organic acid analysis of the urine, is warranted in all patients with unexplained hypotonia and that plasma flavins should be measured in patients with riboflavin responsive MADD in whom sequencing of the relevant genes fails to detected a mutation.

Acknowledgements The authors wish to thank M. Turkenburg for technical assistance, and F.M. Vaz and R.C.M. Hennekam for valuable input and discussions.

Open Access This article is distributed under the terms of the Creative Commons Attribution Noncommercial License which permits any noncommercial use, distribution, and reproduction in any medium, provided the original author(s) and source are credited.

\section{References}

Antozzi C, Garavaglia B, Mora M et al. (1994) Late-onset riboflavinresponsive myopathy with combined multiple acyl coenzyme A dehydrogenase and respiratory chain deficiency. Neurology 44:2153-2158

Capo-chichi CD, Guenat JL, Feillet F, Namour F, Vidailhet M (2000) Analysis of riboflavin and riboflavin cofactor levels in plasma by HPLC. J Chrom B 739:219-224

Dipti S, Childs A, Livingston JH, Aggarwal AK, Miller M, Williams C, Crow YJ (2005) Brown-Vialetto-Van Laere syndrome; variability in age at onset and disease progression highlighting the phenotypic overlap with Fazio-Londe disease. Brain Dev 27:443-446

Duran M, Dorland L, Van den Berg IET et al. (1997) The ethylmalonic acid syndrome is associated with deranged sulfur aminoacid metabolism leading to urinary excretion of thiosulfate and sulfothiocysteine. 8th Int Congress IEM,Vienna, Abstract 048

Goodman SI (1981) Organic aciduria in the riboflavin-deficient rat. Am J Clin Nutr 34(11):2434-2437

Green P, Wiseman M, Crow YJ, et al. (2010) Brown-Vialetto-Van Laere syndrome, a pontobulbar palsy with deafness, is caused by mutations in c20orf54. Am J Hum Genet 12;86(3):485-489

Gregersen N, Andresen BS, Pedersen CB, Olsen RK, Corydon TJ, Bross P (2008) Mitochondrial fatty acid oxidation defectsremaining challenges. J Inherit Metab Dis 31(5):643-657

Leshner RT (1981) Riboflavin deficiency-a reversible neurodegenerative disease. Ann Neurol 10:294-295

Olsen RK, Olpin SE, Andresen BS et al (2007) ETFDH mutations as a major cause of riboflavin-responsive multiple acyl-CoA dehydrogenation deficiency. Brain 130(8):2045-2054

Powers HJ (2003) Riboflavin (vitamin B2) and health. Am J Clin Nutr 77:1352-1360 
Rhead WJ, Wolff JA, Lipson M et al (1987) Clinical and biochemical variation and family studies in the multiple acyl-CoA dehydrogenation disorders. Pediatr Res 21:371-376

Sathasivam S (2008) Brown-Vialetto-Van Laere syndrome. Orphanet J Rare Dis 3:9

Spaan AN, IJlst L, van Roermund CW, Wijburg FA, Wanders RJ, Waterham HR (2005) Identification of the human mitochondrial FAD transporter and its potential role in multiple acylCoA dehydrogenase deficiency. Mol Genet Metab 86(4):441447

Tanaka K, Mantagos S, Genel M, Seashore MR, Billings BA, Baretz BH (1977) New defect in fatty acid metabolism with hypoglycaemia and organic aciduria. Lancet ii:986-987
Tiranti V, Viscomi C, Hildebrandt T et al. (2009) Loss of ETHE1, a mitochondrial dioxygenase, causes fatal sulfide toxicity in ethylmalonic encephalopathy. Nat Med 15:200-205

Van Hove JL, Grünewald S, Jaeken J et al (2003) D, L-3hydroxybutyrate treatment of multiple acyl-CoA dehydrogenase deficiency (MADD). Lancet 361(9367):1433-1435

Ventura FV, Costa CG, Struys EA et al (1999) Quantitative acylcarnitine profiling in fibroblasts using $\left[\mathrm{U}^{13} \mathrm{C}\right]$ palmitic acid: an improved tool for the diagnosis of fatty acid oxidation defects. Clin Chim Acta 281(1-2):1-17

Yamamoto S, Inoue K, Ohta KY et al. (2009) Identification and functional characterization of rat riboflavin transporter 2. J Biochem 145:437-443 